\title{
High Pressure Structural Transitions in Kr Clathrate-Like Clusters
}

\author{
Daniel J. Arismendi-Arrieta ${ }^{\S}$, Ales Vítek ${ }^{\dagger}$, and Rita Prosmiti ${ }^{\S *}$ \\ §Instituto de Física Fundamental (IFF-CSIC), CSIC, Serrano 123, 28006 Madrid, Spain \\ † IT4Innovations National Supercomputing Center, VSB-Technical University of Ostrava, 70833 \\ Ostrava, Czech Republic \\ E-mail: rita@iff.csic.es;Phone:+34-91-561-6800
}

*To whom correspondence should be addressed 


\begin{abstract}
Classical thermodynamic properties of a size-specific Kr clathrate-like cluster, modeled by an $a b$ initio and a semiempirical $\mathrm{Kr}$-water potential, were computed from parallel-tempering Monte Carlo simulations. The temperature and pressure dependence of the cluster's heat capacity was studied, and phase diagrams were constructed using a multiple-histogram method. By associating the heat capacity (maxima) and the Pearson correlation coefficient (minima/maxima) values with the phase transitions, attempts were made to identify such changes to particular cluster structures. Various isomers were computed by local optimizations of the interaction enthalpy at a set of randomly selected configurations at each temperature-pressure grid point. Their energy distributions and relative abundances were then employed to assign the observed phase transitions of the cluster. It was found that the structural changes at high pressures are related to $\mathrm{Kr}$ clathrate-like cages, such as those of the sI, sII and sH hydrates, as well as a new one at higher pressures, formed by tetragons and pentagons. Such transitions, at low temperatures and as pressure increases, are related to the topology of the intermolecular interactions, that are getting accessible by the sampling in the MC simulations, through the employed volume model.
\end{abstract}

\title{
Introduction
}

Simulations of cluster systems can be a challenging and computational demanding task, as the quality and sampling of the underlying potential energy and free-energy surfaces are essential to reliably determine both ground state and finite temperature/pressure properties. As with other finite systems, heterogeneous water clusters have received considerable attention as models for investigating the behavior of aqueous environments in the condensed phase. In particular, most of the studies on neutral clusters of single atom/molecule solutes in water clusters have been motivated by using such clusters as models to understand better properties of clathrate hydrates, ${ }^{1-7}$ or clathrate-like hydration structures at the transition from the liquid to solid phase. ${ }^{8,9}$ High pressure studies are also very important and informative for the understanding of the principal properties of 
such clathrates, ${ }^{10,11}$ and the general features of their formation and dissociation.

Depending on the size of the encapsulated gas molecules, most clathrates form cubic (sI and sII) or hexagonal sH crystal structures. For a fundamental understanding of the size effect on the crystal structure, gas hydrate systems with the simplest guests, have been intensively studied, with the noble gases to serve as prototypes for other guest molecules, e.g. $\mathrm{Ne}$ for $\mathrm{H}_{2}, \mathrm{Ar}$ for $\mathrm{O}_{2} / \mathrm{N}_{2}$, and $\mathrm{Kr}$ for methane. ${ }^{12,13}$ As expected, the large rare gas atoms, such as $\mathrm{Ar}, \mathrm{Kr}$ and $\mathrm{Xe}$, are enclathrated in sII and sI clathrates, respectively, at modest gas pressures, less than $1 \mathrm{GPa}$, and then, at higher pressures they transform to $\mathrm{sH}$ ones. In addition to the classical clathrate structures transitions to two new clathrate structures, structure T (sT) and filled-ice structure $\mathrm{O}(\mathrm{sO})$, have been observed at high pressure in X-ray, neutron diffraction, ${ }^{14}$ and Raman scattering studies. ${ }^{15}$ In the case of $\mathrm{Kr}$ hydrates both experimental studies, ${ }^{14,15}$ have observed that the sII structure transforms initially to the $\mathrm{sI}$, then to $\mathrm{sH}$, and finally to the $\mathrm{sO}$ structure, although there is a disagreement between the two different sets of experiments on the pressure range of these transitions.

Motivated by experimental and theoretical studies on rare-gas clathrate hydrates ${ }^{10,14-26}$ we compute thermodynamic properties of specific size clusters. From our simulations we can obtain not only the ground state properties, but also "phase transition" behavior for such clusters. Of particular interest to us are the phase change properties of the cluster at low temperatures as the pressure increases. Computational studies on the phase diagram of such noble gas hydrate systems will contribute to our understanding, providing insights regarding the formation, and pressure transitions of some selective host water networks, that are able to leverage experimental efforts. Thus, in this article, we report isothermal-isobaric (NPT) parallel tempering Monte Carlo (PTMC) simulations at various temperature and pressure values for the $\mathrm{Kr}\left(\mathrm{H}_{2} \mathrm{O}\right)_{20}$ cluster. Our choice for the number of water molecules, forming the cluster under study, corresponds to the small $\left(5^{12}\right)$ polyhedral cavity, that is the common building block in all sI, sII and sH clathrate structures, as well as to the medium $\left(4^{3} 5^{6} 6^{3}\right)$ cage of the sH structure (see Fig. 1).

Determination of thermodynamic properties requires an extensive sampling of the system configuration space that strongly related to the topology of the underlying potential energy surface 


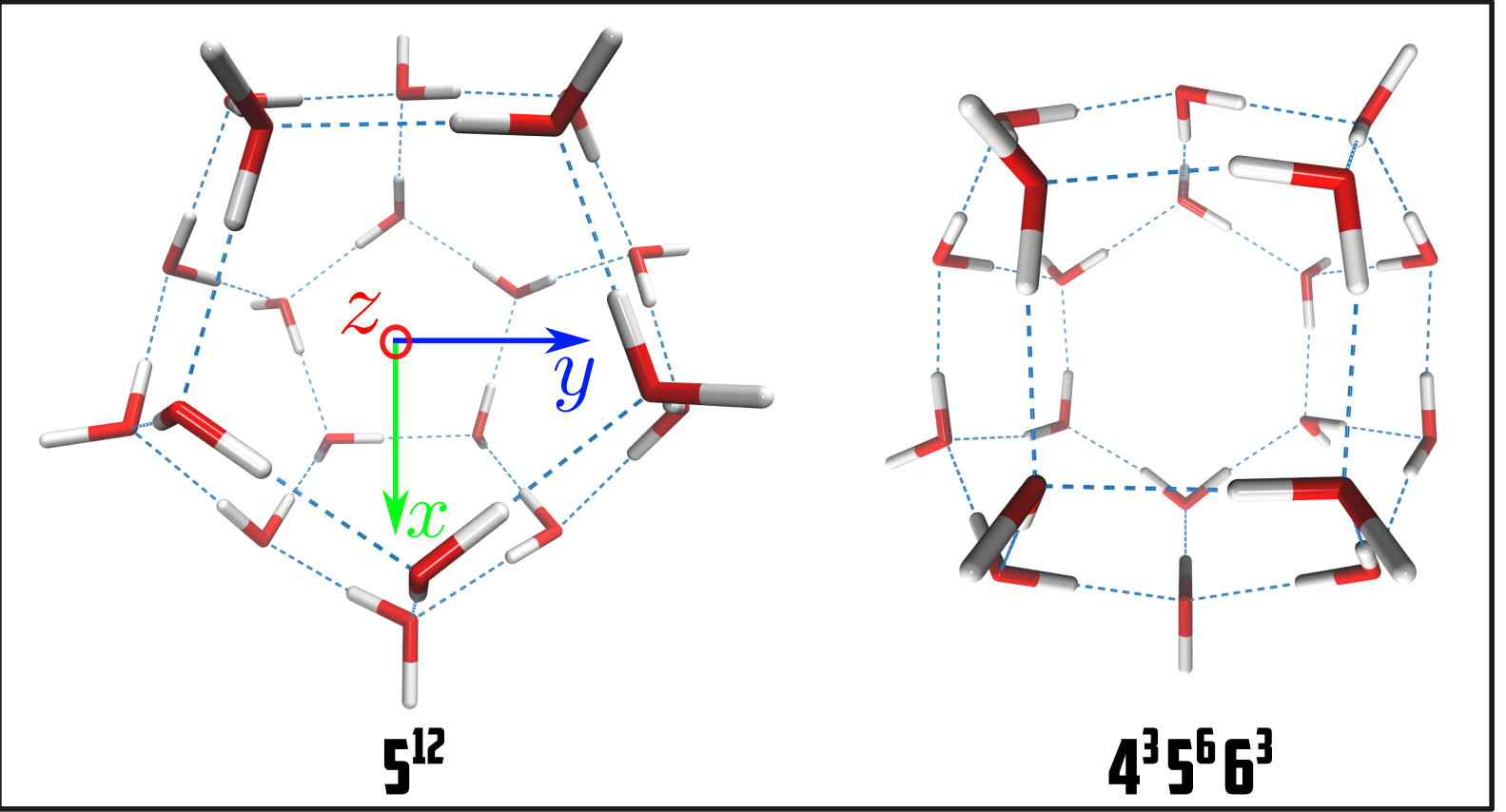

Figure 1: The $5^{12}$ and $4^{3} 5^{6} 6^{3}$ cages.

(PES), and is usually limited by the available computational technology. First-principles on-thefly simulations based on density functional theory methods (including dispersion corrections) for the electronic calculations can be considered as an accurate approach for the potential model, although for systems of quite limited size. ${ }^{27}$ Thus, model potentials based on less computationally demanding force fields have been employed usually for the description of water interaction. ${ }^{28-32}$ Here, we choose the TIP4P/ice water model ${ }^{33}$ as it has been found to give the best description of the phase diagram of the water and its polymorphs in the solid state, as well as the coexistence lines in clathrate hydrate systems. ${ }^{30,34,35}$ For the $\mathrm{Kr}$-water interaction we used two different models based on semiempirical and ab initio procedures, as there is also an underlying interest to explore their reliability and their key role in the determination of thermodynamic properties and phase diagram calculations. We should point out that we associate heat capacity and Pearson correlation coefficient peaks for the cluster with solid-to-liquid like and solid-to-solid like phase changes. Although, current extrapolations from clusters to bulk phase transitions rely on assumptions, the output of such comparison could reveal trends for identifying essential interactions needed for a reliable description of the extended systems. ${ }^{36}$ 
The contents of the remainder of the article are organized as follows: In section 2 we define the potentials models chosen for the study of the cluster and describe out computational methodology; in section 3 we present thermodynamic and structural results and discuss the performance of each $\mathrm{Kr}$-water interaction; in section 4 we summarize our findings and list some concluding remarks.

\section{Computational Methodology}

\section{Model Potential}

The potential energy consists of two terms, water-water and rare gas-water interactions.

$$
V_{t o t}=\sum_{w=1}^{20} \sum_{w^{\prime}>w}\left(V_{w-w^{\prime}}+V_{K r-w}\right)
$$

In this work, we use the TIP4P/ice $\operatorname{model}^{33}$ for the water-water interactions $\left(V_{w-w^{\prime}}\right)$, and two different models for the $\mathrm{Kr}$-water $\left(V_{K r-w}\right)$; one is semiempirical, and the other is the ab initio potential for the $\mathrm{Kr}-\mathrm{H}_{2} \mathrm{O}$ interaction by Makarewicz from Ref. ${ }^{37}$ The semiempirical potential expressions include pair-wise interactions, combining electrostatic terms between the 3-point charges centered on the M-site and $\mathrm{H}$ atoms of the $\mathrm{H}_{2} \mathrm{O}$ molecules, and with van der Waals (vdW) interactions between the $\mathrm{Kr}$ and $\mathrm{O}$ atom of water molecules. It is expressed as,

$$
V_{K r-w}=\sum_{i} \sum_{j>i} 4 \varepsilon_{i j}\left[\left(\frac{\sigma_{i j}}{r_{i j}}\right)^{12}-\left(\frac{\sigma_{i j}}{r_{i j}}\right)^{6}\right]
$$

where, $i$ and $j$ summation runs over $\mathrm{Kr}$ and all $\mathrm{O}, \mathrm{H}$, M-site centers on each $\mathrm{H}_{2} \mathrm{O}$ molecule, respectively. The cross interaction between $\mathrm{Kr}$ and $\mathrm{O}$ atoms is described by the standard LorentzBerthelot combination rules between unlike atoms, $\varepsilon^{i j}=\left(\varepsilon^{i i} \varepsilon^{j j}\right)^{1 / 2}$ and $\sigma^{i j}=\left(\sigma^{i i}+\sigma^{j j}\right) / 2$, with the Lennard-Jones parameters, $\sigma$ and $\varepsilon$, between similar type atoms ${ }^{21,33}$ summarized in Table 1.

The ab initio $\mathrm{Kr}-\mathrm{H}_{2} \mathrm{O}$ surface is based on an analytical many-body (up to 4-body) form, with each term being representing by sums of Morse-type products, which has been corrected asymp- 
Table 1: Semiempirical Force Field Parameters Used in This Work.

\begin{tabular}{cccc}
\hline Atom/Site $($ molecule $)$ & $\mathrm{q}[\mathrm{e}]$ & $\sigma[\AA]$ & $\varepsilon[\mathrm{kJ} / \mathrm{mol}]$ \\
\hline $\mathrm{O}$ (water) & 0.0 & 3.1668 & 0.882159 \\
$\mathrm{H}($ water $)$ & +0.5897 & 0.0 & 0.0 \\
$\mathrm{M}($ water $)$ & -1.1794 & 0.0 & 0.0 \\
\hline $\mathrm{Kr}$ & 0.0 & 3.599 & 1.616 \\
\hline
\end{tabular}

totically by introducing an additional dispersion term. This expression has been fitted to CCSD(T) data refined at their complete basis set limit, and the corresponding 42 parameters are given in Ref. ${ }^{37}$ This potential includes the anisotropy of the $\mathrm{Kr}-\mathrm{H}_{2} \mathrm{O}$ interaction, and in the upper panel of Fig. 2 we plot the ab initio potential curves as a function of the $\mathrm{Kr}-\mathrm{O}$ distance, $R$, in comparison with the corresponding semiempirical one. One can see that the most attractive geometry for the $\mathrm{Kr}-\mathrm{H}_{2} \mathrm{O}$ interaction correspond to planar configuration, namely M1 (see dashed line in Fig. 2), while a saddle point, namely S3 (see solid line in Fig. 2), has an out of plane configuration with the $\mathrm{Kr}$ atom in an almost perpendicular position to the $\mathrm{H}_{2} \mathrm{O}$ plane comparable with the one predicted by the semiempirical pair-wise PES. Thus, based on the ab initio and semiempirical $\mathrm{Kr}-\mathrm{H}_{2} \mathrm{O}$ interactions, we choose to show the potential curves for the $\mathrm{Kr}$ atom with 5 water molecules forming a pentagon, common in both cavities, (see middle panel) as a function of the $\mathrm{Kr}$ to center of mass of the water molecules distance, $\mathrm{z}^{+}$, and with 20 water molecules forming a $5^{12}$ cage (see lower panel) as a function of the $\mathrm{z}$ distance between $\mathrm{Kr}$ atom and center of mass of the cage (see Fig. 1). We can see certain differences between the semiempirical and ab initio potential curves, corresponding at their well-depths and positions, as well as in the repulsive part of them, that are getting larger as the size of the system increases. In particular, in the case of the $5^{12}$ cage, the $\mathrm{Kr}$ atom could be inside or outside the cavity with the high barriers to correspond to the interaction energy for the "on-the-surface" position of the $\mathrm{Kr}$ atom (see lower panel of Fig. 2). As the $\mathrm{Kr}$ atom approaches the surface of the cage, the interaction is getting much more repulsive using the semiempirical potential than the ab initio one, with the values for the barriers to be much higher in this case. Thus, such characteristics of the potential are expected to affect the MC sampling, specially at high pressure conditions. 

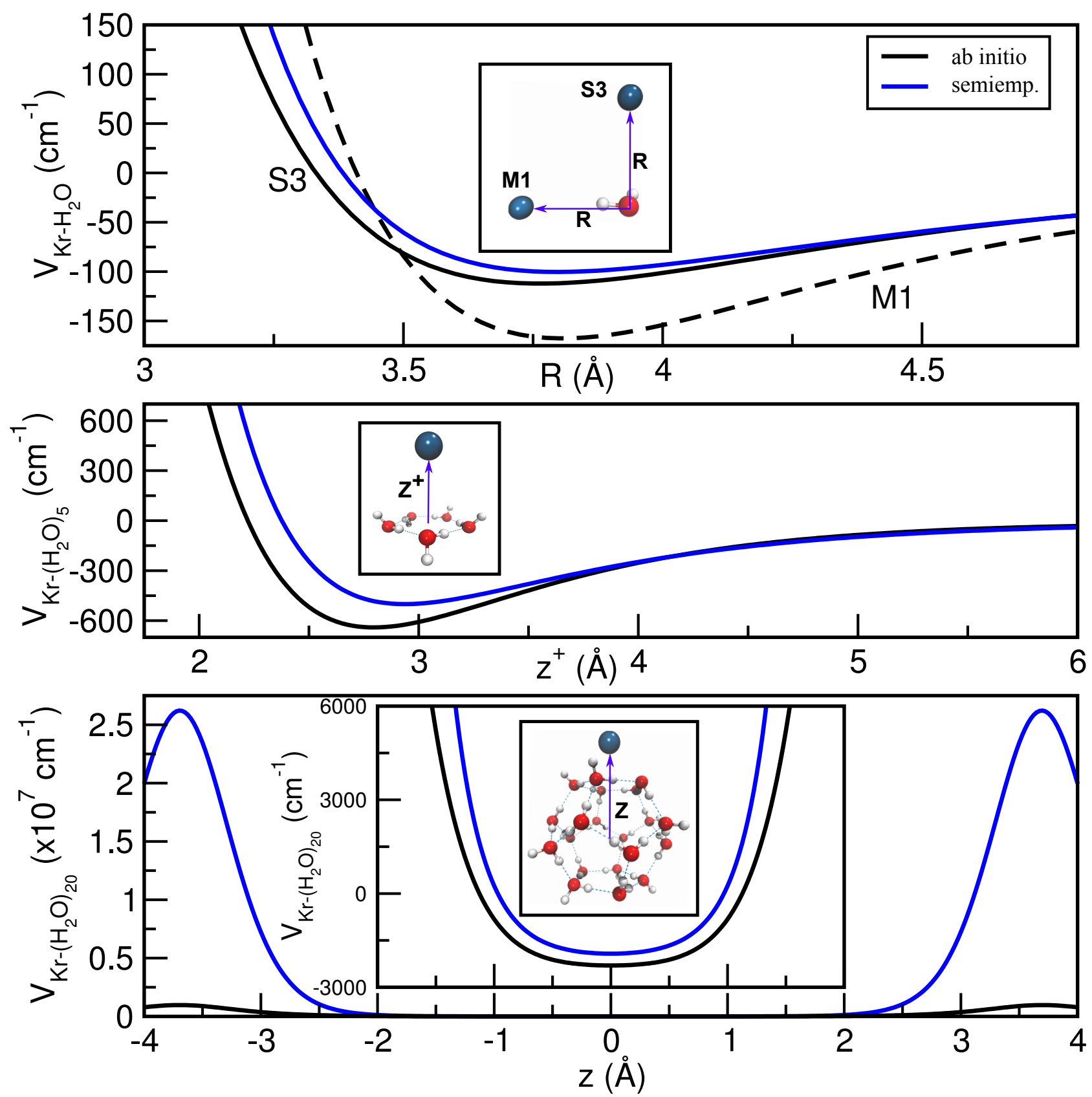

Figure 2: Ab initio and semiempirical potential curves for the $\mathrm{Kr}-\mathrm{H}_{2} \mathrm{O}$ (upper panel), the $\mathrm{Kr}-$ $\left(\mathrm{H}_{2} \mathrm{O}\right)_{5}$ (middle panel), and the $\mathrm{Kr}-\left(\mathrm{H}_{2} \mathrm{O}\right)_{20}$ (lower panel) interactions (see text). 


\section{Parallel-tempering Monte Carlo Computations}

Generally, one has to resort into smart and accelerated Monte Carlo (MC) sampling techniques in order to extract thermodynamical averages from free energy landscapes. In this vein, the PTMC method has been derived ${ }^{38,39}$ to achieve an efficient sampling of systems that have rugged energy landscapes, with a large number of close local minima. The general idea is to simulate $\mathscr{M}$ replicas of the system for a specific ensemble, each of them at a different thermodymanic conditions (temperature and pressure), and allowing completely configuration exchange between different systems.

Here, an isothermal-isobaric (NPT) ensemble is considered, and the residual part of the mean value of a dynamic parameter, $F$, if it depends on the system configuration, $q$, only through the interaction energy and system volume, is expressed at particular temperature $T$ and pressure $P$ as,

$$
\langle F\rangle_{T, P}=\frac{\int_{V} \int_{E^{\mathrm{int}}} F\left(E^{\mathrm{int}}, V\right) \exp \left(-\frac{E^{\mathrm{int}}+P V}{k_{\mathrm{B}} T}\right) \Omega\left(E^{\mathrm{int}}, V\right) \mathrm{d} E^{\mathrm{int}} \mathrm{d} V}{Z_{T, P}},
$$

where $\Omega\left(E^{\text {int }}, V\right)$ is the classical density of states and

$$
Z_{T, P}=\int_{V} \int_{E^{\mathrm{int}}} \exp \left(-\frac{E^{\mathrm{int}}+P V}{k_{\mathrm{B}} T}\right) \Omega\left(E^{\mathrm{int}}, V\right) \mathrm{d} E^{\mathrm{int}} \mathrm{d} V
$$

is the system configuration integral, $V$ is the volume of the system, $E^{\text {int }}$ is the interaction energy of the system, and $k_{\mathrm{B}}$ is the Boltzmann constant.

A two-dimensional parallel-tempering algorithm has been used to accelerate the convergence of the present NPT MC simulations. ${ }^{40}$ Periodic swaps of configurations and volumes between replicas of the simulated system have been used. Only neighboring systems are selected, maintaining in this way the exchange probability sufficiently high, using the standard Metropolis-Hasting criterion ${ }^{41}$ for the acceptance probability for translational, rotational and volume changes. The MC simulations are carried out in parallel for 23 pressure and 18 temperatures values, ranging between $3 \mathrm{kPa}$ to $10 \mathrm{GPa}$ and 30 to $1000 \mathrm{~K}$, respectively. Note that the upper temperature limit is chosen to 
warranty a rapid converge in the PTMC calculations. For both grid values a non-uniform exponential increase of spacing is used for an efficient sampling of the system configurations, given that energy and volume fluctuations are smaller at low temperatures and high pressures. The external pressure is approximated by a hard-wall spherical container volume model of a variable radius, ${ }^{42}$ with an upper limit for it set to $200 \AA$ for controlling the volume growth at low pressures and high temperatures. In total, 414 replicas of the system are simulated in parallel, about $5 \times 10^{7} \mathrm{MC}$ steps are performed for reaching each system at thermodynamic equilibrium, and another $5 \times 10^{7} \mathrm{MC}$ whole-cluster and volume moves are performed for each system replica by collecting data for the final analysis every $50^{\text {th }} \mathrm{MC}$ step to avoid correlations.

\section{Computation of Specific Thermodynamic Averages}

For the evaluation of the ensemble averages (see Eq. 3) a sufficiently accurate estimate of the 2D density of states, $\Omega\left(E^{\mathrm{int}}, V\right)$, is required. This represents a rather tough task consuming the more computer time, and usually, the grid of (414 in this case) simulated temperatures and pressures is strongly limited to the computational demands. Thus, we adopt the multiple-histogram (MH) approach, ${ }^{43,44}$ as it has been recently implemented, ${ }^{45}$ for these calculations at an acceptable computational cost. In a first step, the collected data from the above series of NPT PTMC simulations are used to produce 414 energy-volume histograms, using exponential spacing grids each of them of 1000 different energies and 2000 different volumes. In turn, the 414 energy-volume histograms are used in an iterative scheme to obtain the values of interest at any thermodynamic condition (not included in the particular simulations). In this way, using the 2D MH approach, the 2D density of states, $\Omega\left(E^{\mathrm{int}}, V\right)$, is obtained from 160000 temperatures-pressure points, instead of the initial 414 ones from the NPT PTMC simulations. Once the 2D density of states is calculated, the final calculation of the ensemble averages from Eq. 3 is simply the computation of the 2D integral.

In this way, selected thermodynamic averages, such as enthalpy, heat capacity and Pearson correlation coefficient for energy and volume, are calculated. For interpreting phase changes in the $\mathrm{Kr}\left(\mathrm{H}_{2} \mathrm{O}\right)_{20}$ cluster, we used the constant-pressure heat capacity, $C_{P}$, along temperature, and 
the Pearson correlation coefficient for energy and volume, $\rho_{E^{\text {int }}, V}$, along the pressure/temperature. Here, the residual part of the constant-pressure heat capacity is considered as,

$$
C_{P}^{\mathrm{res}}=\frac{1}{k_{\mathrm{B}} T^{2}}\left[\left\langle\left(H^{\mathrm{int}}\right)^{2}\right\rangle-\left\langle H^{\mathrm{int}}\right\rangle^{2}\right],
$$

where $H^{\text {int }}=E^{\text {int }}+P V$, while the Pearson correlation coefficient for volume and energy is given by,

$$
\rho_{E^{\mathrm{int}, V}}=\frac{\left\langle E^{\mathrm{int}} V\right\rangle-\left\langle E^{\mathrm{int}}\right\rangle\langle V\rangle}{\sigma_{E^{\mathrm{int}}} \sigma_{V}},
$$

with $\left\langle E^{\text {int }}\right\rangle$ and $\langle V\rangle$ denoting the mean values and $\sigma_{E^{\text {int }}}$ and $\sigma_{V}$ being the variances of the interaction energy and volume, respectively.

Phase diagrams are constructed by plotting the cluster heat capacity and the Pearson correlation coefficient as a function of temperature and pressure. For finite systems the phase changes are not so sharp transitions as in bulk, and cover a wide region of temperatures and pressures (coexistence regions). However in a simplified way, we represent them by curves, calling them coexistence curves, that correspond to the maxima of the heat capacity, $C_{P}^{\mathrm{res}}$, along temperature, and to minima and maxima of the Pearson correlation coefficient for interaction energy and volume, $\rho_{E^{\text {int }}, V}$, along the pressure and temperature, respectively. In particular, the $\rho_{E^{\mathrm{int}}, V}$ minima (corresponding to strong anti-correlation between interaction energy and volume of the system) along the pressure axis are used to detect different solid-like phases, while its maxima (indicating a strong correlation between interaction energy and volume of the system), as well as the maxima of the $C_{P}^{\text {res }}$, along temperature axis correspond to evaporative regions of the cluster.

\section{Results and Discussion}

\section{Phase Diagrams}

In Fig. 3 we show temperature-pressure phase diagrams for the $\operatorname{Kr}\left(\mathrm{H}_{2} \mathrm{O}\right)_{20}$ cluster calculating from PTMC simulations using the $a b$ initio (see upper panel) and semiempirical (see lower panel) po- 
tentials for the Kr-water interaction and the TIP4P/ice model for the water-water one. As it can be seen, the temperature-pressure plane is divided into several regions corresponding to different phases of the cluster. The corresponding coexistence curves (as we mentioned above simplified representations of broader coexistence regions for finite size systems) are obtained by calculating numerically the maxima of the heat-capacity as $\left[\partial C_{P}^{\text {res }} / \partial T\right]_{P}=0$ (see black dotted-lines), the maxima (see red dotted-lines) and minima (see blue dotted-lines) of the Pearson correlation coefficient as $\left[\partial \rho_{E_{\text {int }}, V} / \partial T\right]_{P}=0$ and $\left[\partial \rho_{E^{\text {int }}, V} / \partial P\right]_{T}=0$, respectively. At low temperatures the cluster is solid-like, although as the temperature increases the cluster becomes liquid-like, while at even higher temperatures a liquid-like to gas-like transition occurs. In particular, in both phase diagrams we observe evaporation of the water molecules in the cluster for $\mathrm{T}>300 \mathrm{~K}$, the cluster melting for $\mathrm{T}>250 \mathrm{~K}$ and $\mathrm{P}>100 \mathrm{kPa}$, and the evaporation of the $\mathrm{Kr}$ atom at $\mathrm{T}<150 \mathrm{~K}$ and $\mathrm{P}<100 \mathrm{kPa}$. One should note that for the cluster evaporation both coexistence curves obtained either from the heat capacity (black dotted-lines) or from the Pearson correlation coefficient (red dotted-lines) coincide up to pressure values of $10 \mathrm{MPa}$, while for higher pressures deviations are found. This is due to the flatness on the temperature dependence of the maxima of the heat capacity, with the coexistence curve calculated using the Pearson correlation coefficient to represent better the liquid-like to gas-like transition.

The dissociation curve of the Kr-water clathrate systems has been measured by Dyadin et al. ${ }^{16,46}$ up to $1.5 \mathrm{GPa}$, and they have observed transitions from sII to sI and to sH structures. Such transitions have been also found from X-ray diffraction studies ${ }^{14}$ at ambient temperatures and at pressures of 0.3 and $0.6 \mathrm{GPa}$, respectively, in addition to the formation of sO structure at $1.8 \mathrm{GPa}$, which it is then decomposed at $3.8 \mathrm{GPa}$. More recently, Raman scattering measurements ${ }^{15}$ are also available for the transitions from the sII to sI to $\mathrm{sH}$ and $\mathrm{sO}$ structures at $0.45,0.75$ and $1.8 \mathrm{GPa}$, respectively. They observed that the $\mathrm{sO}$ structure of the $\mathrm{Kr}$ hydrate exists at least up to $5.2 \mathrm{GPa}$ at $296 \mathrm{~K}$, while a new phase behavior at $1.0 \mathrm{GPa}$ has been attributed to an increase in the large-cage occupancy. ${ }^{15}$ Further, in a recent theoretical study by Subbotin et al. ${ }^{21}$ the sII to sI phase transition has been predicted at $0.17 \mathrm{GPa}$. One can see that the available studies are all in accord regarding 

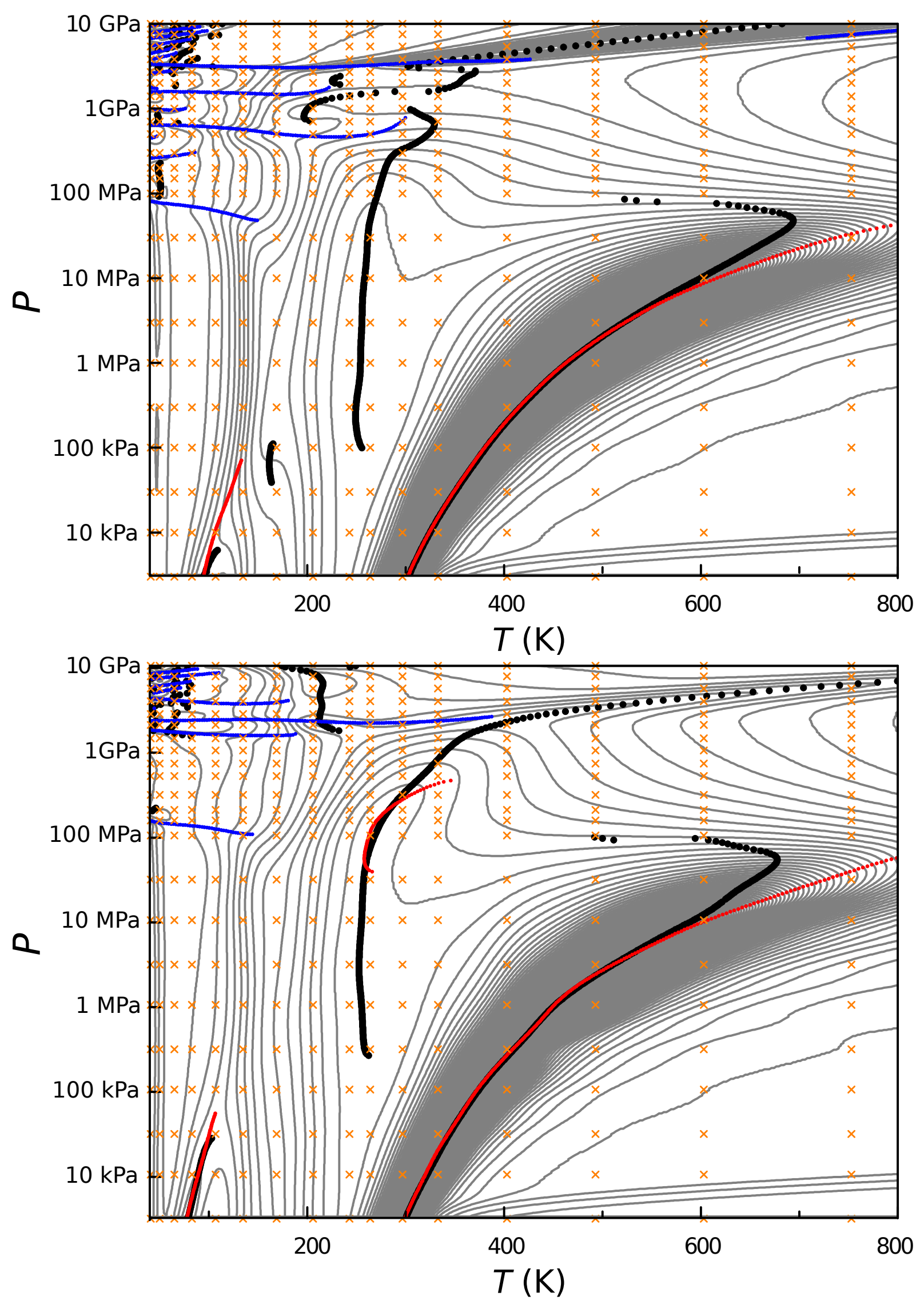

Figure 3: Pressure-temperature phase diagrams for the $\operatorname{Kr}\left(\mathrm{H}_{2} \mathrm{O}\right)_{20}$ cluster computed from the PTMC simulations using the ab initio (upper panel) and semiempirical (lower panel) potential energy surfaces for the $\mathrm{Kr}-\mathrm{H}_{2} \mathrm{O}$ interaction, and the TIP4P/ice model for the water-water ones (see text). Cross symbols indicate the $(T, P)$ grid points used in the present PTMC simulations. 
the formation of the sI, $\mathrm{sH}$ and $\mathrm{sO}$ structures, although with discrepancies at the pressure values, where the corresponding transitions occur.

Here, we should point out that the curve of the heat capacity maxima (solid-to-liquid like coexistence curve) obtained by using the ab initio $\mathrm{Kr}-\mathrm{H}_{2} \mathrm{O}$ interaction shows a discontinuous behavior at pressure values higher than $0.7 \mathrm{GPa}$. As we mentioned above the heat capacity surface is getting very flat, and very non significant maximum changes its own position. This is also the case for the $\mathrm{Kr}$ evaporations curve at low temperature and pressures, which also shows similar behavior for the ab initio potential. By comparing the behavior of the phase diagrams at low temperatures and high pressure regime $(\mathrm{P}>50 \mathrm{MPa})$, corresponding to solid-to-solid like structural changes of the cluster, one can see that the Pearson energy-volume correlation coefficient profiles show structural transformations at similar pressures, for both type of Kr-water interactions, while some others take place at different values. In particular, using the ab initio $\mathrm{Kr}$-water interaction (see upper panel of Fig. 3) structural changes are expected at pressures around $100 \mathrm{MPa}, 0.3,0.7,1.0,1.5$, and 3.8 GPa, while for the semiempirical model (see lower panel of Fig. 3) transitions are predicted for pressure values just above $100 \mathrm{MPa}, 1.5,2.0$, and 3.8 GPa.

In Figure 4 we show the pressure dependence of the interaction energy and the mean simulation volume for few selected temperatures for the semiempirical potential. As the cluster configuration sampling during the NPT simulations is governed by the cluster enthalpy, it is expected that at low-pressure the cluster interaction energy dominates with low-energy configurations being preferred, while at high-pressures small volume configurations may be favored over the ones with lower energy. Consequently, such behavior is expected to produce a smooth step in the curves of energy/volume as a function of pressure in finite-size systems, indicating the region where a transformation of the cluster structures takes place. One can see such sudden drop-off in both energy and volume curves in Fig. 4 particularly at lower temperatures, e.g. $T=62,104$ and $166 \mathrm{~K}$, indicating solid-to-solid like transformations. The transition pressures (around 0.15, 1.5, 2.2 and $3.8 \mathrm{GPa}$ ) are almost independent of the temperature, although at $T=294 \mathrm{~K}$ the drop-off disappears as the cluster is in liquid-like phase. Also, as it was expected the cluster energy and volume are 


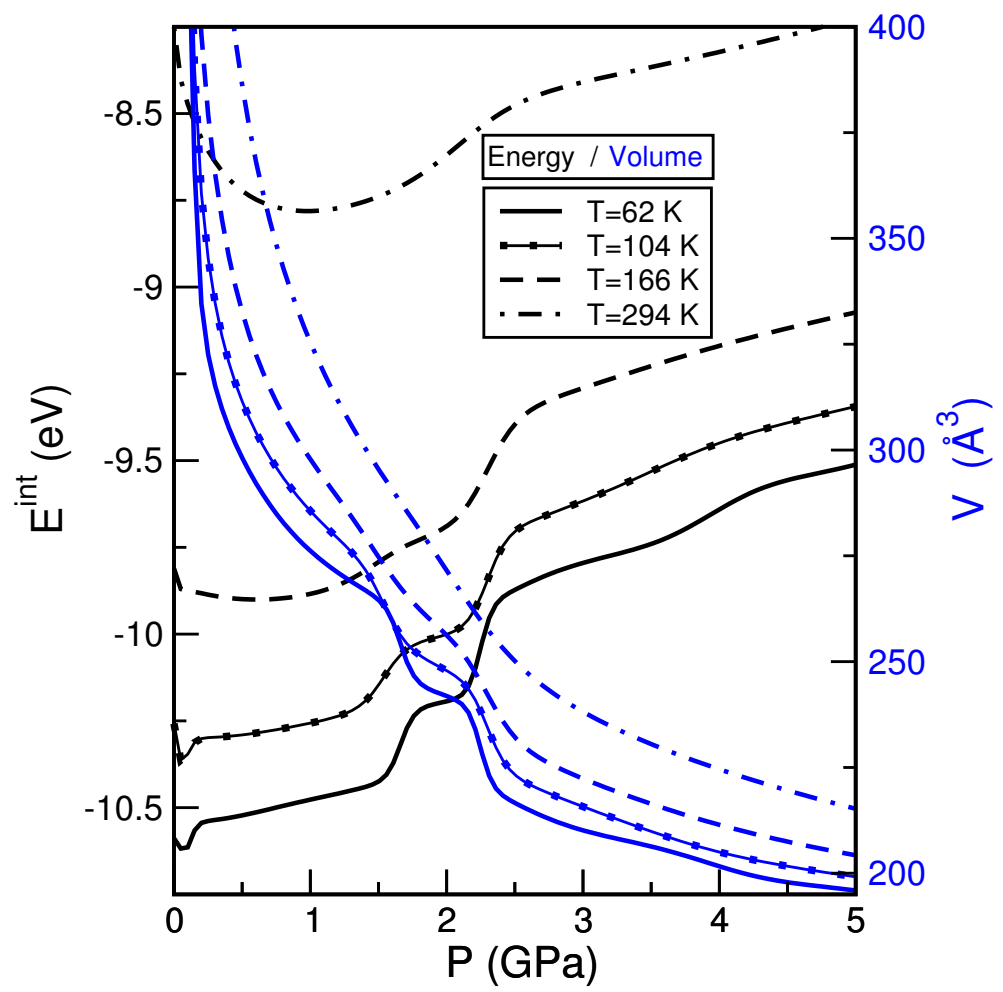

Figure 4: Interaction energy and mean volume dependences of the pressure at the indicated temperatures $\mathrm{T}$ for the $\mathrm{Kr}\left(\mathrm{H}_{2} \mathrm{O}\right)_{20}$ using the semiempirical $\mathrm{Kr}$-water potential model.

strongly anti-correlated, due to the competition between potential energy and the pressure-volume term in the enthalpy form. Such behavior is clearly reflected to the minimum values of the Pearson correlation coefficient, $\rho_{E^{\text {int }}, V}$, as are shown in Fig. 3, and below we will discuss on the characterization of these observed transitions.

\section{Structural Isomers and Specific Phase Transitions}

For analyzing the structures under different $T, P$ conditions, local enthalpy optimizations are also carried out for 96 randomly selected configurations for each $T, P$ point from the NPT PTMC simulations, for all temperature grid values in the 62-294 K range, and all grid pressure values from 0.3 MPa up to $10 \mathrm{GPa}$, in total 16416 configurations for each potential model. Such minimizations are performed via the simulated annealing method ${ }^{47}$ for a NPT statistical ensemble using a probability weight of $\exp \left(-\frac{\left(E^{\mathrm{int}}+P V\right)}{k_{\mathrm{B}} T}\right)$ for sampling the cluster configurations and volumes, with starting temperature of $5 \mathrm{~K}$ and final one of $0.01 \mathrm{~K}$. Current temperature has been decreased every $50^{\text {th }}$ 


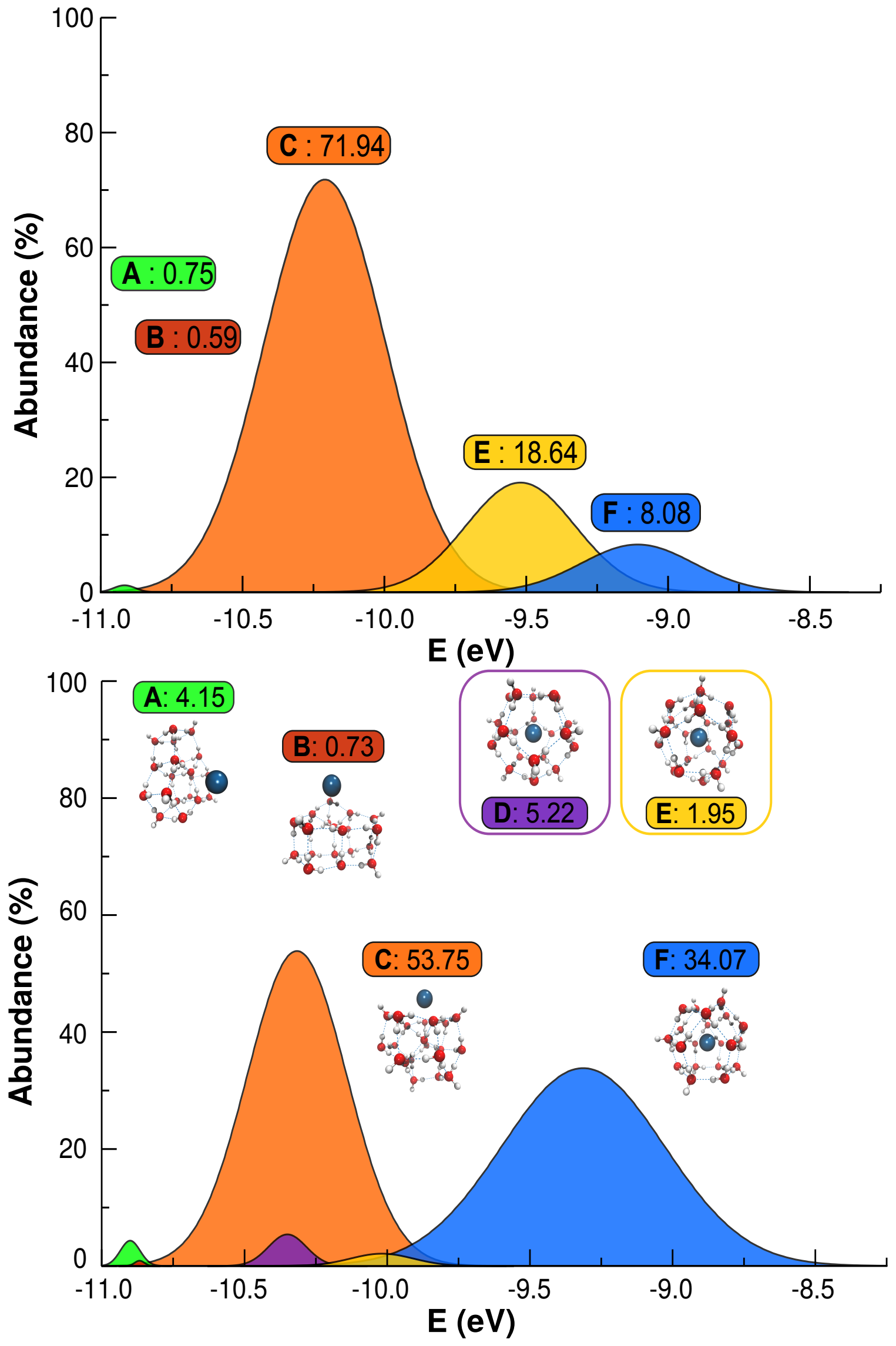

Figure 5: Relative abundance of the six structufal isomers of the $\mathrm{Kr}\left(\mathrm{H}_{2} \mathrm{O}\right)_{20}$ cluster obtained by optimized locally cluster enthalpies as a function of the interaction energy, $E^{\text {int }}$ for the ab initio (upper panel) and semiempirical (lower panel) Kr-water potential. The corresponding isomeric structures are also displayed. 
MC step by a factor of 0.999 . We should note that the fast cooling and the low initial temperature were adopted so that an optimization to the nearest local minimum for each configuration to be performed. We carried out such analysis for both $a b$ initio and semiempirical model potentials and we observed isomeric structures such as the ones shown in Figure 5 (see lower panel), namely A, B C, D, E, and F. We should note that for the ab initio potential (see upper panel in Fig. 5) we only found isomeric structures corresponding to $\mathrm{A}, \mathrm{B} \mathrm{C}, \mathrm{E}$ and $\mathrm{F}$ configurations, with $\mathrm{C}$ being the predominal one for low pressure $(80.69 \%)$ and $E$ for high pressures $(12.89 \%)$, while for the semiempirical model (see lower panel in Fig. 5) we observed all the above mentioned structures.

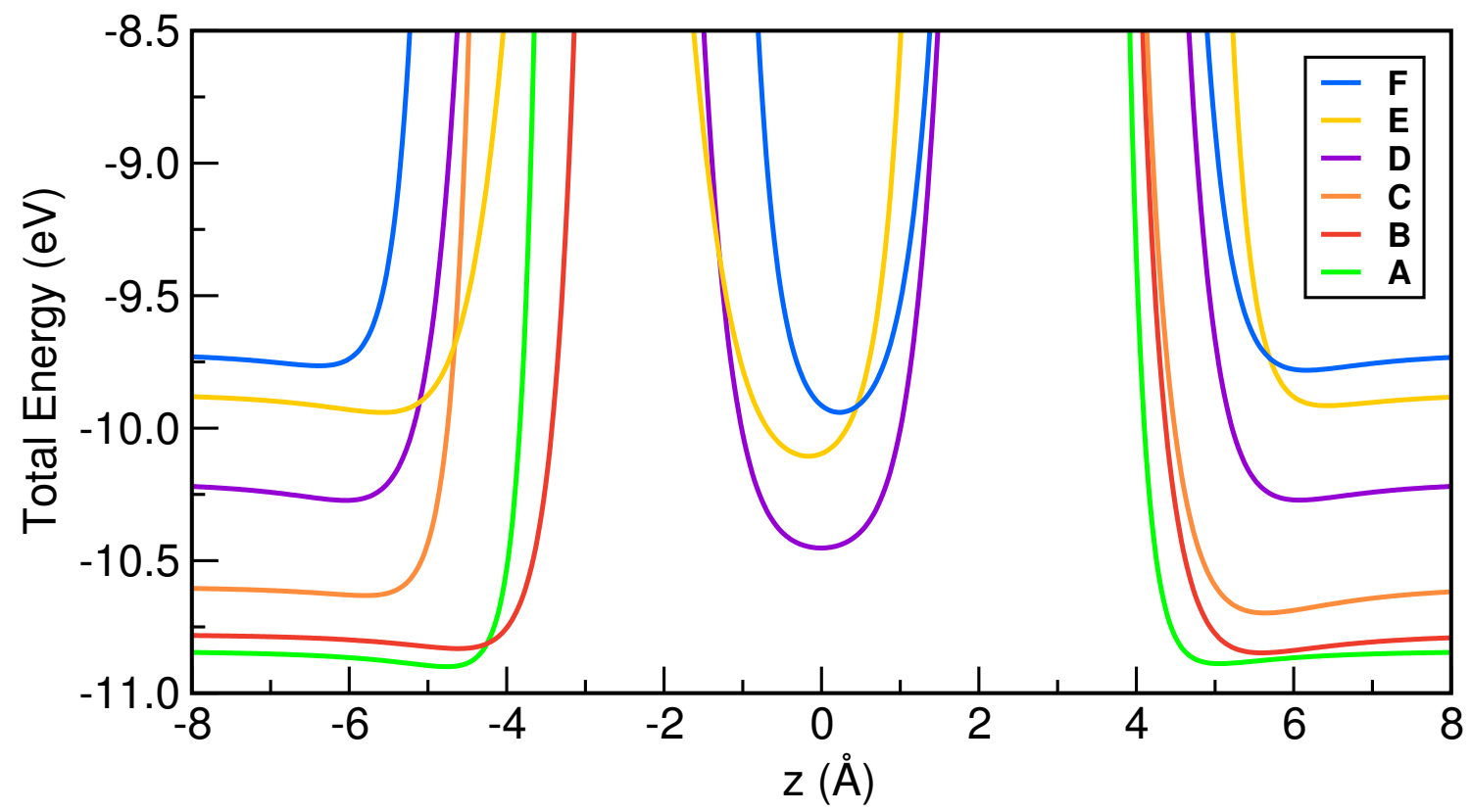

Figure 6: Total potential energy (see Eq. (1)) scans using the TIP4P/ice for the water-water and the semiempirical Kr-water models for each of the six isomeric structure observed for the $\mathrm{Kr}\left(\mathrm{H}_{2} \mathrm{O}\right)_{20}$ along the $z$-axis (see text).

As the characterization of the structures are performed according to their interaction energy at each $(T, P)$ point of the grid, we thus first discuss the energy distribution of the structures. In Figure 5 we show a plot containing the different isomeric structures observed classifying them according to their abundances and energies. We choose to represent such distributions by Gaussian functions fitted to the energy histograms for each isomeric structure. One can see that at lower energies we observed the structures A and B (with a percentage of 4.15 and $0.73 \%$, respectively), 
corresponding to an edge-sharing pentagonal prism, and a near "all-surface" for the water cluster, respectively, with the $\mathrm{Kr}$ atom outside. We should point out that the population of the structures is highly depending on the $(T, P)$ points as well as the $(T, P)$ grid intervals (see Fig. 3). In Figure 5 we present relative abundance from 171 temperature-pressure systems, and thus, by including the whole range of temperature and pressure values the total abundance, especially of those isomers at low pressures, such as A and B ones, should be increased. Next, the structure C: a cage-1 structure for the water with the $\mathrm{Kr}$ atom outside, appears, and is present for a quite wide energy range from around -10.8 to $-10.2 \mathrm{eV}$. This isomeric structure is also the most abundant one for the semiempirical potential with $53.75 \%$, while at energies around $-10.3,-10.0$, and $-9.5 \mathrm{eV}$ we obtained the clathrate-like isomeric structures, $\mathrm{D}, \mathrm{E}$, and $\mathrm{F}$ with the $\mathrm{Kr}$ atom at the interior of a $5^{12}$ structure (see also left panel of Fig. 1), an irregular cage formed from tetragons, pentagons, and hexagons (similar to the $4^{3} 5^{6} 6^{3}$ one shown in the right panel of Fig. 1), and a cage formed from tetragons and pentagons, respectively. The abundance of the $\mathrm{D}$ structure is $5.22 \%$, for the $\mathrm{E}$ is $1.95 \%$, while the $\mathrm{F}$ structure is the predominant one with $34.07 \%$ at energies of around $-9.25 \mathrm{eV}$. So, at low energies/pressures open/on-surface structures are found, while closed/clathrate-like ones appear at high energies/pressures. We should also note the energy overlapping for different structures, due to the competition between the potential and $P V$ terms, at energies around $-10.8 \mathrm{eV}$ and between -10.5 and $-9.75 \mathrm{eV}$. In Figure 6 we present scans of the total potential energy corresponding to A, $\mathrm{B}, \mathrm{C}, \mathrm{D}, \mathrm{E}$ and $\mathrm{F}$ isomeric structures along the $\mathrm{z}$-axis of the $\mathrm{Kr}\left(\mathrm{H}_{2} \mathrm{O}\right)_{20}$ cluster. One can see that the curves of the A, B and C open/on-surface structures show shallow minima at energies between -11.0 to $10.5 \mathrm{eV}$, while the minima of the $\mathrm{D}, \mathrm{E}$, and $\mathrm{F}$ close/clathrate-like structures are at energies above $-10.5 \mathrm{eV}$, sampling different configurations.

In Figure 7 we show the pressure dependence of each isomers's abundance at specific temperature values over all included systems, while in Figure 8 we display 2D contour plots of the isomeric structure abundances, together with the coexistence curves corresponding to the maxima of the heat capacity (black dotted lines) and to the minima of the Pearson correlation coefficient (blue dotted lines), as shown in the lower-panel of Fig. 3, at low-ambient temperatures and all pressure values 


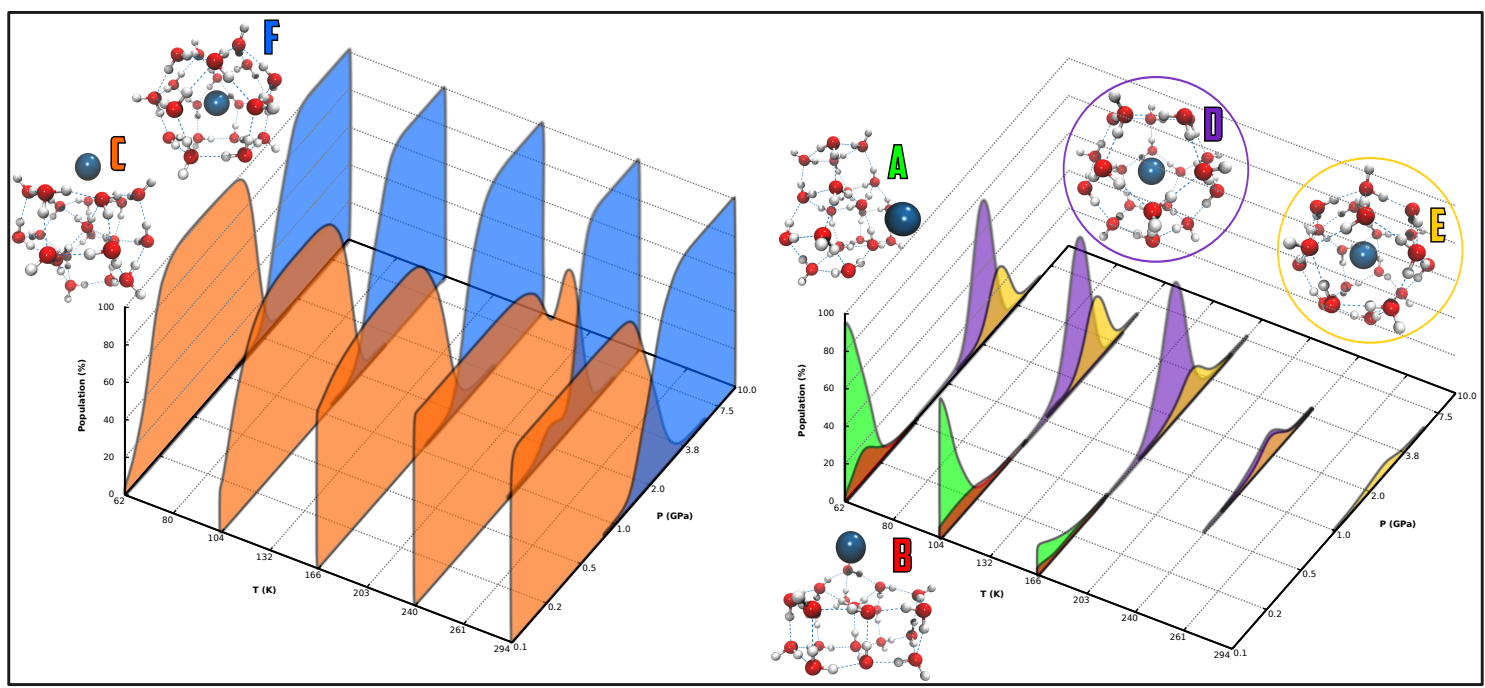

Figure 7: Relative abundance of the indicated structural isomers (see A, B, C, D, E and F in the inset plots) of the $\mathrm{Kr}\left(\mathrm{H}_{2} \mathrm{O}\right)_{20}$ cluster obtained by optimized locally cluster enthalpies as a function of pressure at specific temperature values using the semiempirical $\mathrm{Kr}$-water potential.

studied. One can clearly see the $(P, T)$-region where each structural transition occurs. In particular, at low temperatures and as the pressure increases we observed the $\mathrm{A} \longrightarrow \mathrm{B} \longrightarrow \mathrm{C}, \mathrm{C} \longrightarrow \mathrm{D}, \mathrm{D} \longrightarrow \mathrm{E}$, and $\mathrm{E} \longrightarrow \mathrm{F}$ transitions near at $0.2,1.5,2.2$, and 3.8 GPa, respectively. All these solid-to-solid like transitions are clearly marked by the minima of the Pearson correlation coefficient (see blue dotted lines in Figure 8). Further, at ambient temperatures one can see the $\mathrm{C} \longrightarrow \mathrm{F}$ transition above 2.0 GPa, while the solid-like to liquid-like transition of the F structure, as it is shown by the corresponding coexistence curve at $T>210 \mathrm{~K}$ and $P>2.0 \mathrm{GPa}$ (see black dotted line in Figure 8). This latter transition appears by a continuous rearrangement mainly of the hydrogens' positions in the clathrate-like structure, similar to the movements of the Rubik's cube.

Certainly the accuracy of the results depends on the accuracy of the potential model deployed. Here, we used pairwise host-guest interactions from semiempirical and ab initio data for the $\mathrm{Kr}$ water dimer. We found that the ab initio-based interactions couldn't predict the regular D clathratelike structure. As the main structure-determining factor for these clathrate hydrates, especially at high pressure, is the guest-host lattice interaction, such finding indicates qualitatively differences between the two PESs. A simple correction of the pairwise host-guest interaction, using MP2 or $\operatorname{CCSD}(\mathrm{T})$ results for the dimer potential energy surface should provide an upper bound limit for the 


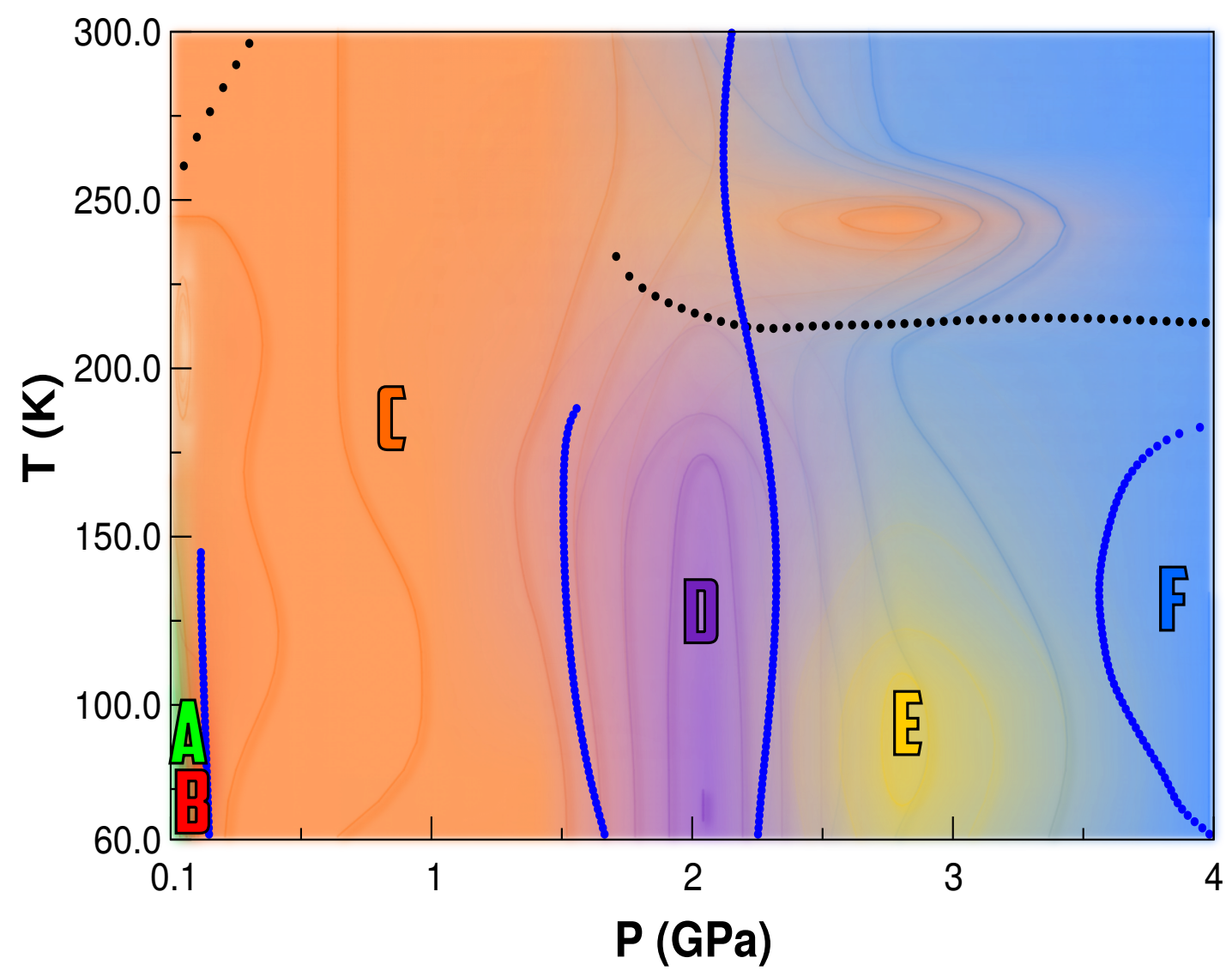

Figure 8: Contour plot of the abundance of each isomeric structure (A in green, $\mathrm{B}$ in red, $\mathrm{C}$ in orange, D in violet, E in yellow, and $\mathrm{F}$ in blue color) observed for the $\mathrm{Kr}\left(\mathrm{H}_{2} \mathrm{O}\right)_{20}$ at $P T$-plane. The coexisted curves corresponding to the maxima of heat capacity (black dotted lines) and minima of Pearson correlation coefficient (blue dotted lines) are also shown.

hydrate binding energies, that leds to the instability of the regular clathrate structure. Moreover, density functional methods used to predict interaction energies between similar rare-gas host and guest species, have been found to overestimate the binding at least in small gas phase systems. ${ }^{26}$ However, even if this overbinding has been found less pronounced in the extended systems, ${ }^{48}$ specialized treatments of such weak host-guest interactions, for example, on the basis of semi-local or hybrid functionals including dispersion and optimized exchange-correlation density functionals for condensed water systems ${ }^{49,50}$ or optimized noble-gas-water pair potentials via Monte Carlo simulations, ${ }^{51}$ might be instructive and lead to gain further insights.

As we mentioned above, experimental data from X-ray diffraction ${ }^{14}$ and Raman scattering ${ }^{15}$ 
measurements are available for Kr hydrate up to pressures of almost 5.2 GPa. Given the discrepancies it has been argued ${ }^{15}$ that transitions at high pressure are related with the occupancy of the large-cage, and theoretical simulations ${ }^{28}$ have provided some support, suggesting that pressure tends to stabilize higher occupancy. However, here we have treated the small cage, and as we point out further work is needed to explore the effects of guest-host interactions. In addition, and in connection with the nature of the interaction, the effect of the volume definition model for simulations at NPT ensembles on finite non-periodic systems should be also considered, e.g. by implementing more axiomatic volume models, such as those using the convex polyhedron that circumscribed all atoms in the cluster, ${ }^{52}$ and/or the "quantum volume" occupied by the electronic charge density. ${ }^{53}$

\section{Summary and conclusions}

Parallel-tempering isothermal-isobaric Monte Carlo simulations were carried out at temperature values from 30 to $1000 \mathrm{~K}$ and pressures between $3 \mathrm{kPa}-10 \mathrm{GPa}$ for the $\mathrm{Kr}\left(\mathrm{H}_{2} \mathrm{O}\right)_{20}$ cluster. We employed pairwise interactions for representing the water-water and $\mathrm{Kr}$-water potential terms using the TIP4P/ice model, and a semiempirical or an ab initio-based potential, respectively. Structural and thermodynamic properties, such as cluster energy and enthalpy, constant-pressure heat capacity, volume, and Pearson correlation coefficient for energy and volume, were calculated, and were used to construct temperature-pressure phase diagrams. By focusing our analysis at low temperatures, we observe structural changes corresponding to solid-solid like transitions, that at low/medium pressure values (up to $1.5 \mathrm{GPa}$ ) were assigned to open/on-surface isomeric structures, such as the "all-surface" and cage-1 like structures for the water cluster with the Kr atom outside, while as pressure increases (up to $10 \mathrm{GPa}$ ) closed/clathrate-like isomers, like the regular $5^{12}$, as well as other irregular new ones, occur. In particular, structural changes from outside-to-inside structures are observed at $1.5 \mathrm{~Pa}$ to regular $5^{12}$ structures, then at $2.2 \mathrm{GPa}$ to irregular, similar to the $4^{3} 5^{6} 6^{3}$ cages, while at $3.8 \mathrm{GPa}$ structures formed by tetragons and hexagons are also found. We show that qualitatively differences between the semiempirical and $a b$ initio-based pairwise 
guest-host interactions, as well as, through the MC configurations sampling, the volume definition model for non-periodic finite-size systems, could directly affect the formation of specific isomeric structures, especially under high pressures. Therefore, the rational route to follow is, to improve first the description of the interaction, and to check the effect of the volume definition in the simulations. On the one hand, further work in this direction employing specialized first-principles treatments for determining the weak host-guest interactions under pressure should be performed. In this way, one could obtain an estimate of the systematic error and evaluate the accuracy of the interaction potential from finite size to extended state systems. On the other hand, the volume definition model for non-periodic finite-size systems should also be examined by implementing different more axiomatic models, such as reservoir and extended Lagrangian approaches, in the PTMC simulations for a better description of general shape finite systems that are caged. The output of such studies could reveal trends for a more reliable representation of the interactions, which could then serve for an accurate and complete analysis from finite size systems (clusters) to periodic 3D networks. Unfortunately, no experimental data are yet available for Kr-water clusters of any size for a direct comparison. However, we hope that our theoretical findings will stimulate and guide such experimental investigations. Meanwhile our future efforts should focus to extend the present simulations to larger size clusters, although theoretically still tractable. Such clusters correspond to the cavities of the sI, sII, sH, sO, and sT type structures of the Kr clathrate hydrates aiming, as a first step, to an indirect comparison with experimental observations available for these systems, and thus to a better quantitative description of the underlying interactions.

\section{Acknowledgement}

The authors thank support MICINN grants No. FIS2014-51933-P, COST Actions CM1204 (XLIC) and CM1405(MOLIM), the Ministry of Education, Youth and Sports of the Czech Republic from the National Programme of Sustainability (NPU II) project "IT4Innovations excellence in science LQ1602" and from the Large Infrastructures for Research, Experimental Development and Innovations project "IT4Innovations National Supercomputing Center - LM2015070". The calculations 
were performed on the HPC resources of the IT4Innovations National Supercomputing Center, VSB - Technical University of Ostrava (grants no. OPEN-7-48, DD-15-32 and DD-16-16), and the Centro de Calculo (IFF/SGAI-CSIC).

\section{References}

(1) Yoo, S.; Kirov, M. V.; Xantheas, S. S. Low-Energy Networks of the T-Cage $(\mathrm{H} 2 \mathrm{O})_{24}$ Cluster and Their Use in Constructing Periodic Unit Cells of the Structure I (sI) Hydrate Lattice. $J$. Am. Chem. Soc. 2009, 131, 7564-7566.

(2) Hartke, B. Methane-Water Clusters Under Pressure: Are Clathrate Cages Optimal Clusters? J. Chem. Phys. 2011, 130, 024905-024913.

(3) Holden, G. L.; Freeman, D. L. Monte Carlo Investigation of the Thermodynamic Properties of $\left(\mathrm{H}_{2} \mathrm{O}\right)_{n}$ and $\left(\mathrm{H}_{2} \mathrm{O}\right)_{n} \mathrm{H}_{2}(n=2-20)$ Clusters. J. Phys. Chem. B 2011, 115, 4725-4744.

(4) Yoo, S.; Xantheas, S. S. Handbook of Computational Chemistry; Springer, 2012; pp 761-792.

(5) Vítek, A.; Ofiala, A.; Kalus, R. Thermodynamics of Water Clusters Under High Pressures. A Case Study for $\left(\mathrm{H}_{2} \mathrm{O}\right)_{15}$ and $\left(\mathrm{H}_{2} \mathrm{O}\right)_{15} \mathrm{CH}_{4}$. Phys. Chem. Chem. Phys. 2012, 14, 15509-15519.

(6) Colognesi, D.; Celli, M.; Ulivi, L.; Xu, M.; Bačić, Z. Neutron Scattering Measurements and Computation of the Quantum Dynamics of Hydrogen Molecules Trapped in the Small and Large Cages of Clathrate Hydrates. J. Phys. Chem. A 2013, 117, 7314-7326.

(7) Valdés, A.; Arismendi-Arrieta, D. J.; Prosmiti, R. Quantum Dynamics of Carbon Dioxide Encapsulated in the Cages of the sI Clathrate Hydrate: Structural Guest Distributions and Cage Occupation. J. Phys. Chem. C 2015, 119, 3945-3956.

(8) Bowron, D. T.; Filipponi, A.; Roberts, M. A.; Finney, J. L. Hydrophobic Hydration and the Formation of a Clathrate Hydrate. Phys. Rev. Lett. 1998, 81, 4164-4167. 
(9) Ashbaugh, H. S.; Asthagiri, D.; Pratt, L. R.; Rempe, S. B. Hydration of Krypton and Consideration of Clathrate Models of Hydrophobic Effects from the Prespective of Quasi-Chemical Theory. Biophys. Chem. 2003, 105, 323-338.

(10) Loveday, J. S.; Nelmes, R. J. High-Pressure Gas Hydrates. Phys. Chem. Chem. Phys. 2008, 10, 937-950.

(11) Strobel, T. A.; Somayazulu, M.; Hemley, R. J. Phase Behavior of $\mathrm{H}_{2}+\mathrm{H}_{2} \mathrm{O}$ at High Pressures and Low Temperatures. J. Phys. Chem. C 2011, 115, 4898-4903.

(12) Sloan, E. D.; Koh, C. A. Clathrate Hydrates of Natural Gasess, 3rd ed.; CRC Press, 2007.

(13) Loveday, J. S.; Nelmes, R. J.; Guthrie, M.; Klug, D. D.; Tse, J. S. Transition from Cage Clathrate to Filled Ice: The Structure of Methane Hydrate III. Phys. Rev. Lett. 2001, 87, 215501-215505.

(14) Desgreniers, S.; Flacau, T.; Klug, D.; Tse, J. Dense Noble Gas Hydrates: Phase Stability and Crystalline Structures. Proceedings abstract of CeSMEC conference. Florida, 2003; p 24, March.

(15) Sasaki, S.; Hori, S.; Kume, T.; Shimizui, H. Microscopic Observation and In-Situ Raman Scattering Studies on High-Pressure Phase Transformations of Kr Hydrate. J. Phys. Chem. B 2006, 110, 9838-9842.

(16) Dyadin, Y.; Larionov, Â.; Aladko, E.; Manakov, A.; Zhurko, F.; Mikina, T.; Komarov, V.; Grachev, E. Clathrate Formation in Water-Noble Gas (Hydrogen) Systems at High Pressures. J. Struct. Chem. 1999, 40, 790-795.

(17) Dyadin, Y. A.; Larionov, E. G.; Manakov, A. Y.; Zhurko, F. V.; Aladko, E. Y.; Mikina, T. V.; Komarov, V. Y. Clathrate Hydrates of Hydrogen and Neon. Mendeleev Commun. 1999, 9, 209-210. 
(18) Manakov, A.; Voronin, V.; Kurnosov, A.; Teplykh, A.; Larionov, E.; Dyadin, Y. A. Argon Hydrates: Structural Studies at High Pressures. Dokl. Phys. Chem. 2001, 378, 148-151.

(19) Subbotin, O. S.; Belosludov, V. R. Relationship Between the Dynamic Properties of 1h Ice and Xenon Hydrate and the Interplay of Intramolecular and Intermolecular Vibrations. $J$. Struct. Chem. 2002, 43, 545-551.

(20) Tse, J.; Klug, D.; Zhao, J.; Sturhahn, W.; Alp, E.; Baumert, J.; Gutt, C.; Johnson, M.; Press, W. Anharmonic Motions of $\mathrm{Kr}$ in the Clathrate Hydrate. Nat. Mater. 2059, 4, 917921.

(21) Subbotin, O. S.; Adamova, T. P.; Belosludov, R. V.; Mizuseki, H.; Kawazoe, Y.; Kudoh, J.I.; Rodger, P. M.; Belosludov, V. R. Theoretical Study of Phase Transitions in $\mathrm{Kr}$ and Ar Clathrate Hydrates from Structure II to Structure I Under Pressure. J. Chem. Phys 2009, 131, $114507-114515$.

(22) Papadimitriou, N. I.; Tsimpanogiannis, I. N.; Stubos, A. K.; Martin, A.; Rovetto, L. J.; Peters, C. J. Unexpected Behavior of Helium as Guest Gas in sII Binary Hydrates. J. Phys. Chem. Lett. 2010, 1, 1014-1017.

(23) Belosludov, R. V.; Bozhko, Y. Y.; Subbotin, O.; Belosludov, V. R.; Mizuseki, H.; Kawazoe, Y.; Fomin, V. M. Stability and Composition of Helium Hydrates Based on Ices Ih and II at Low Temperatures. J. Phys. Chem. C 2014, 118, 2587-2593.

(24) Yu, X.; Zhu, J.; Du, S.; Xu, H.; Vogel, S. C.; Han, J.; Germann, T. C.; Zhang, J.; Jin, C.; Francisco, J. S. et al. Crystal Structure and Encapsulation Dynamics of Ice II-Structured Neon Hydrate. Proc. Natl. Acad. Sci. 2014, 111, 10456-10461.

(25) Vitek, A.; Arismendi-Arrieta, D. J.; Rodriguez-Cantano, R.; Prosmiti, R.; Villarreal, P.; Kalus, R.; Delgado-Barrio, G. Computational Investigations of the Thermodynamic Properties of Size-Selected Water and Ar-water Clusters: High-Pressure Transitions. Phys. Chem. Chem. Phys. 2015, 17, 8792-8801. 
(26) Teeratchanan, P.; Hermann, A. Computational Phase Diagrams of Noble Gas Hydrates Under Pressure. J. Chem. Phys. 2015, 143, 154507-1-9.

(27) Wang, J.; Lu, H.; Ripmeester, J. A.; Becker, U. Molecular-Dynamics and First-Principles Calculations of Raman Spectra and Molecular and Electronic Structure of Hydrogen Clusters in Hydrogen Clathrate Hydrate. J. Phys. Chem. C 2010, 114, 21042-21050.

(28) Alavi, S.; Ripmeester, J. A.; Klug, D. D. Stability of Rare Gas Structure H Clathrate Hydrates. J. Chem. Phys. 2006, 125, 104501-104511.

(29) Frankcombe, T. J.; Kroes, G. J. Molecular Dynamics Simulations of Type-sII Hydrogen Clathrate Hydrate Close to Equilibrium Conditions. J. Phys. Chem. C 2007, 111, 1304413052.

(30) Vega, C.; Abascal, J. L. F.; Conde, M. M.; Aragones, J. L. What Ice Can Teach Us About Water Interactions: a Critical Comparison of the Performance of Different Water Models. Faraday Discuss. 2009, 141, 251-276.

(31) Luis, D.; López-Lemus, J.; Mayorga, M.; Romero-Salazar, L. Performance of Rigid Water Models in the Phase Transition of Clathrates. Mol. Simul. 2010, 36, 35-40.

(32) Vega, C.; Abascal, J. L. F. Simulating Water With Rigid Non-Polarizable Models: a General Perspective. Phys. Chem. Chem. Phys. 2011, 13, 19663-19688.

(33) Abascal, J.; Sanz, E.; Fernández, R. G.; Vega, C. A Potential Model for the Study of Ices and Amorphous Water: TIP4P/Ice. J. Chem. Phys. 2005, 122, 234511-1-10.

(34) Vega, C.; Sanz, E.; Abascal, J.; Noya, E. Determination of Phase Space Diagrams via Computer Simulations: Methodology and Applications to Water, Electrolytes and Proteins. J. Phys.: Condens. Matter 2008, 20, 153101-1-38.

(35) Conde, M.; Vega, C. A Simple Correlation to Locate the Three Phase Coexistence Line in Methane-Hydrate Simulations. J. Chem. Phys. 2013, 138, 056101-056103. 
(36) Wales, D. J.; Doye, J. P. K. In Large Clusters of Atoms and Molecules; Martin, T. P., Ed.; Springer Netherlands: Dordrecht, 1996; pp 241-279.

(37) Makarewicz, J. Ab Initio Intermolecular Potential Energy Surfaces of the Water-Rare Gas Atom Complexes. J. Chem. Phys. 2008, 129, 184310-184320.

(38) Swendsen, R. H.; Wang, J.-S. Replica Monte Carlo Simulation of Spin-Glasses. Phys. Rev. Lett. 1986, 57, 2607-2609.

(39) Geyer, C. Computing Science and Statistics, Proceedings of the 23rd Symposium on the Interface, American Statistical Association, New York. 1991; p 156.

(40) Okabe, T.; Kawata, M.; Okamoto, Y.; Mikami, M. Replica-Exchange Monte Carlo Method for the Isobaric-Isothermal Ensemble. Chem. Phys. Lett. 2001, 335, 435-439.

(41) Metropolis, N.; Rosenbluth, A. W.; Rosenbluth, M. N.; Teller, A. H.; Teller, E. Equation of State Calculations by Fast Computing Machines. J. Chem. Phys. 1953, 21, 1087-1092.

(42) Cheng, H.-P.; Li, X.; Whetten, R. L.; Berry, R. S. Complete Statistical Thermodynamics of the Cluster Solid-Liquid Transition. Phys. Rev. A 1992, 46, 791-800.

(43) Ferrenberg, A. M.; Swendsen, R. H. Optimized Monte-Carlo Analysis. Phys. Rev. Lett. 1989, 63, 1195-1198.

(44) Pal, A.; Roy, S. K. Monte Carlo Simulation of the Nematic-Isotropic Transition in an Isothermal-Isobaric Ensemble. Phys. Rev. E 2004, 69, 021709.

(45) Vítek, A.; Kalus, R. Two-dimensional Multiple-Histogram Method Applied to IsothermalIsobaric Monte Carlo Simulations of Molecular Clusters. Comput. Phys. Commun. 2014, $185,1595-1605$.

(46) A. Dyadin, Y.; G. Larionov, E.; V. Mikina, T.; I. Starostina, L. Clathrate Formation in $\mathrm{Kr}-\mathrm{H}_{2} \mathrm{O}$ and $\mathrm{Xe}-\mathrm{H}_{2} \mathrm{O}$ Systems Under Pressures up to 15 kbar. Mendeleev Commun. 1997, 7, 74-76. 
(47) Kirkpatrick, S.; Jr, C. G.; Vecchi, M. Optimization by Simulated Annealing. Science 1983, $220,671-680$.

(48) Cox, S. J.; Towler, M. D.; Alfé, D.; Michaelides, A. Benchmarking the Performance of Density Functional Theory and Point charge Force Fields in Their Description of sI Methane Hydrate against Diffusion Monte Carlo. J. Chem. Phys. 2014, 140, 174703-1-8.

(49) Gillan, M. J.; Alfé, D.; Michaelides, A. Perspective: How Good Is DFT for Water? J. Chem. Phys. 2016, 144, 130901-1-33.

(50) Fritz, M.; Fernández-Serra, M.; Soler, J. M. Optimization of an Exchange-Correlation Density Functional for Water. J. Chem. Phys. 2016, 144, 224101-1-9.

(51) Warr, O.; Ballentine, C. J.; Mu, J.; Masters, A. Optimizing Noble Gas-Water Interactions via Monte Carlo Simulations. J. Phys. Chem. B 2015, 119, 14486-14495.

(52) Calvo, F.; Doye, J. P. K. Pressure Effects on the Structure of Nanoclusters. Phys. Rev. B 2004, $69,125414$.

(53) Cococcioni, M.; Mauri, F.; Ceder, G.; Marzari, N. Electronic-Enthalpy Functional for Finite Systems Under Pressure. Phys. Rev. Lett. 2005, 94, 145501. 


\section{Graphical TOC Entry}

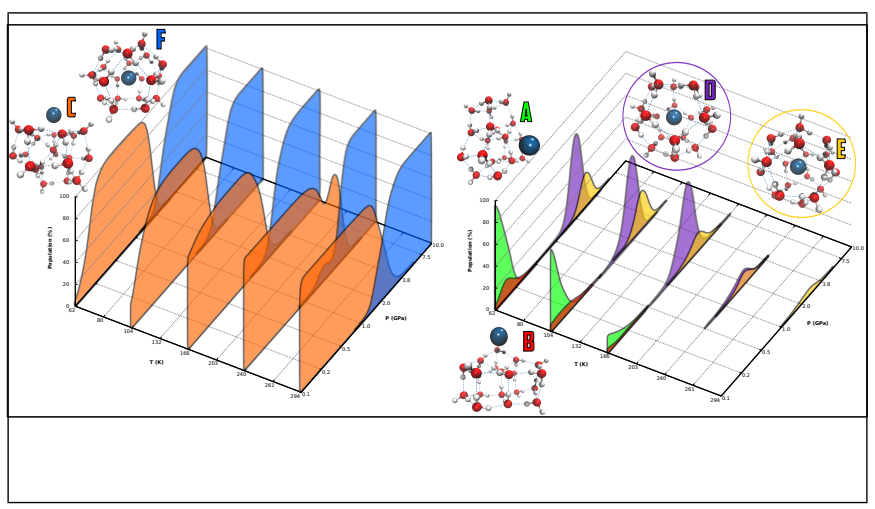

